\title{
Impacts of a Large and Decentralized Telepathology Network in Canada
}

\author{
Guy Paré, Ph.D. * \\ Chair in Information Technology in Health Care \\ HEC Montréal \\ 3000, Côte-Sainte-Catherine Road \\ Montréal (Québec) H3T 2A7 Canada \\ Phone: 1514 340-6812 \\ Fax: 1514 340-6132 \\ Email: guy.pare@hec.ca \\ Julien Meyer, Ph.D. Candidate \\ HEC Montréal \\ Email: julien.meyer@hec.ca \\ Marie-Claude Trudel, Ph.D. \\ HEC Montréal \\ Email: marie-claude.trudel@hec.ca \\ Bernard Têtu, MD \\ Centre Hospitalier Universitaire de Québec \\ Email: bernard.tetu@fmed.ulaval.ca
}

* Corresponding author

Running title: Impacts of a Decentralized Telepathology Network

April 28, 2015 


\begin{abstract}
Background: Telepathology is one of the fast growing segment of the telemedicine field and Canada is recognized as a world leader in this particular domain.

Introduction: We report a benefits evaluation study of a decentralized telepathology network deployed in Eastern Quebec. The project involves 18 hospitals, making it one of the largest telepathology networks in the world.
\end{abstract}

Materials and Methods: We first conducted 43 semi-structured interviews with telepathology users and managers. Hard data on the impacts of the telepathology network (e.g. the number of service disruptions, the average time between initial diagnosis and surgery) was also extracted and analyzed, where available.

Results: Users found the system to be easy to use and the quality of the virtual slides and images was also considered satisfactory by pathologists. A key objective was to provide continuous coverage of intraoperative consultations in hospitals with no pathologist. Our findings show that no service disruptions were recorded in these sites. Surgeons agreed that the use of telepathology helped avoid second surgeries and improved accessibility to care services. Telepathology was also perceived by respondents as having positive impacts on remote hospitals' ability to retain and recruit specialists.

Discussion: The observed benefits should not leave the impression that implementing telepathology is a trivial matter. Indeed, many technical, human and organizational challenges may be encountered.

Conclusions: Telepathology can be highly useful in regional hospitals that do not have a pathologist on site. More research is needed to investigate the challenges and benefits associated with this growing form of telemedicine. 


\section{Introduction}

Telepathology is defined by the American Telepathology Association as "the electronic multimedia communication across a network of pathology-related information, between two or more locations for use-cases between pathologists and/or qualified laboratory personnel, and may include involvement by clinicians and/or patients" [1:5]. This particular form of telemedicine has several applications; it can be used for distant primary diagnoses, expert referrals, quality assurance, and education [2]. For distant diagnoses in the absence of a local pathologist or a telepathology system, slides must be physically sent to another facility, hence delaying the diagnoses. With telepathology, the slides are examined remotely, either statically or dynamically, and diagnoses are swiftly provided. In some particular instances, however, pathology examinations are performed while the patient is still under anaesthetics and undergoing surgery, and the surgeon needs a pathology diagnosis to properly resume his procedure. For these specific examinations, called intraoperative frozen sections, delays are undesirable. When there is no pathologist on site and a surgical procedure requires an intraoperative consultation (IOC), either the patient is transferred to another hospital, a visiting pathologist is called on site, or a procedure is performed in two steps (creating time for the slides to be sent and read elsewhere). Put simply, IOC via telepathology refers to a preliminary diagnosis rendered from macroscopic and/or microscopic images of a frozen tissue section that has been processed rapidly during surgery [3].

Telepathology is currently one of the fast growing segments of the telemedicine field. Indeed, recent figures show that its market is expected to grow from $\$ 2.2$ million in 2013 to nearly $\$ 4.5$ billion in 2018 [4]. It has been implemented in many countries in Europe, Asia and North-America. Canada is recognized as a world leader in this area with several telepathology projects being deployed nationwide. The large geographic size of the country combined with a dispersed population and a shortage of pathologists in several remote regions have contributed to the development of telepathology in Canada [5].

The province of Quebec, where the present study was conducted, is the second most populated province in Canada with its 8.2 inhabitants [6]. In this article we report a benefits evaluation study of the Eastern Quebec telepathology network. The project, which has been initiated with the financial support (CAN\$ 6.2M) of the Quebec government and Canada Health Infoway, currently involves 18 operational sites, making it one of the largest telepathology networks in the world. Of those sites, four have no pathology laboratory, four have a laboratory but no pathologist and there is only one practicing pathologist in four other hospitals. A vast majority of the 48 pathologists involved in this project are concentrated in the teaching medical center located in Quebec City. The telepathology network covers a broad territory of almost $410,000 \mathrm{~km}^{2}$, with a population of more than 1.7 million inhabitants that ranges in density from 0.4 and 9.1 inhabitants per $\mathrm{km}^{2}$. The equipment and software were deployed in late 2010 while clinical activities began in January 2011. Each participating site was equipped with a whole-slide scanner, a macroscopy station and two videoconferencing devices equipped with a drawing tablet. More details about the equipment and software can be found elsewhere [7]. 
While the telepathology network covers the full range of applications mentioned above, its primary objective was to provide IOCs to community hospitals lacking onsite pathologists. In this regard, IOCs are performed on a regular basis; they are originating from 13 of the 18 operational sites and there are seven referral sites. Most IOCs are from breast cancers (sentinel lymph nodes, margin close to breast cancer), lung cancers (bronchial margins, mediastinal lymph nodes), ovarian, pleural, peritoneal, omental lesions, or from stomach and head and neck cancers [7]. As per January 2015, a total of 1733 IOCs were performed via telepathology across the network. The second goal of the project was to achieve substantial gains in terms of speed and quality of surgical services in remote areas. Last, the telepathology network also aimed at facilitating the recruitment and retention of surgeons in remote regions.

Besides its size and scope, another distinctive characteristic of the telepathology network in Eastern Quebec is the decentralized organization of the IOC service [8]. Indeed, in contrast with telepathology projects elsewhere in Canada and abroad, there is no single responding site to which community hospitals without pathologists turn for pathology services. Each requesting hospital is responsible for identifying one or more partner institutions with which it can sign service agreements. It was also initially decided that the teaching hospital located in Quebec City would be most helpful at providing expert opinions to pathologists working alone and to act as a safety net for regional hospitals with temporary interruption of IOC service.

In short, the main objective of this study is to provide a deeper understanding of the actual and perceived impacts of the telepathology network on clinicians, patients, and the overall organization and delivery of care services in Eastern Quebec.

\section{Materials and Methods}

From a methodological standpoint, we adopted a mixed-methods approach. We first conducted a series of semi-structured interviews with telepathology users (pathologists, surgeons, laboratory technologists) and managers. Interview guides containing the specific issues to be discussed with each group of respondents were developed and used during the data collection phase. The data collection process continued until theoretical saturation [9] was reached; i.e. when additional data no longer contributed anything new. All in all, 43 interviews, 34 with clinicians and 11 with managers, were conducted during seven field visits.

The interviews were tape-recorded and then transcribed verbatim, producing about 1,110 pages of transcripts. These were then coded and analyzed using a grid of success indicators for telepathology projects developed by a pan-Canadian committee of experts [10]. Open coding was also performed so that any unforeseen themes that emerged from the data was also captured [11]. Data analysis was performed using the NVivo software package. Ethics approvals for this study were obtained from the lead author's academic institution.

Second, pre-post quantitative data on the impacts of the telepathology network (e.g. the number of service disruptions, the average time between initial diagnosis and surgery) was 
collected and analyzed, where available. These data came from the clinical-administrative information systems (e.g. OPERA, Omnitech, paper registers, etc.) of the hospitals involved.

\section{Results}

Prior to system deployment, the clinicians we interviewed had some concerns about system reliability. As one of the surgeons said, "I had no problem with the remote pathologist reading the slides, but I was a little concerned that the technology wouldn't work." Following some initial technical problems during the start-up period, the system and technological infrastructure deployed in the various sites proved to be highly reliable. One of the most serious technical problems caused a 30-minute delay during a planned IOC, which had no consequences for the patient. As for system friendliness, most users had only positive comments to share. Overall, they found the software to be both simple to learn and easy to use.

The quality of the virtual slides and images generated by the system was also considered satisfactory by pathologists. A quality assurance investigation conducted by a small group of pathologists showed a 98\% concordance rate between the 104 diagnoses made on the frozen material of the IOC cases and the corresponding final diagnoses rendered on paraffin material [12]. For the more complex or ambiguous cases often seen in hematopathology or pulmonary pathology, however, many pathologists mentioned that they couldn't have a precise diagnosis with a digital slide. When faced with complex cases, they would rather follow the "normal" procedure as one pathologist reports below:

"I send all my consultations on complex cases by mail for the simple reason that the first thing that a pathologist working remotely will do is ask for special colorations or additional ones."

As mentioned earlier, the main objective of the telepathology network was to provide continuous coverage of IOC in regional hospitals that do not have a pathologist onsite. A disruption in pathology services can have several negative impacts on surgeries which can either be cancelled or performed in two separate interventions because it takes time for the requested diagnosis to arrive by mail. A service disruption can also cause some anxiety "on a human level, particularly if the patient is not up to a second surgery," said one pathologist. Alternatively, a more invasive surgical intervention may be performed to avoid a second procedure, or the patient may be transferred to another hospital that has a pathologist on staff.

Based on the data that we extracted from administrative systems, it is clear that telepathology assured continuous IOC coverage. In fact, no service disruptions were recorded in the requesting sites that used telepathology. As one surgeon noted:

"When our pathologist left, we already had telepathology in place, so we had no cause for concern. And my patients didn't 
need to wait four weeks for a visiting pathologist to come to our hospital. As far as I know, not a single surgery was cancelled since telepathology was introduced."

In one of the requesting sites, we also measured the average wait time between initial diagnosis (leading to the decision to operate) and the day of the resulting surgery. The available data was mainly for core needle biopsies and endoscopic biopsies. These analyses are performed on permanent sections prepared after processing in formalin and paraffin and not on frozen section material processed during a surgery. They must be interpreted within 24 to 48 hours because they are used to determine whether cancer is present or not and to schedule surgery. A total of 16 cases were found in the pre-period (when the analysis were performed by an onsite pathologist) and 12 cases were found in the post-period (when the analysis were performed remotely by a pathologist using telepathology). The average wait time for all these cases declined from 77 days (pre) to 62 days (post). Based on these results, it appears that performing diagnoses at a distance does not add to the wait time, as might have been previously thought. Despite such encouraging sign, more data needs to be collected and analyzed to confirm this result.

Importantly, the level of satisfaction with regard to IOC via telepathology was high among the surgeons we met. One explanation is that the wait time added by using telepathology is considered acceptable: "Telepathology adds about five or six minutes per report compared with when our pathologist was on-site," one said. The data we extracted from administrative systems at one of the requesting sites showed that IOCs via telepathology took an average of 21 minutes compared with an average of 15 minutes for IOCs performed on site. This result was also supported by the quality assurance investigation [7].

The second objective of this project was to reduce delays and increase the quality of client services in remote regions, in particular by avoiding the need for second surgeries and the transfer of patients to urban centres. While no reliable hard data was available with regard to these particular indicators, all the surgeons we interviewed agreed that the use of the technology helped avoid second surgeries and improved accessibility to care. As they see it, the added value associated with telepathology is mainly due to improved quality of care, as one explained:

"Without telepathology, there may be two surgeries instead of one for the same patient, meaning more invasive surgeries than we would have performed if we had received the opinion of a pathologist in a timely manner. This means a lot for our patients, and it can also make all the difference in the surgery itself."

According to the pathologists we met, telepathology helps prevent medical errors as one mentioned:

"I send a request for a second opinion to a colleague in Ottawa, for example, and he quickly confirms the diagnosis. This way, you 
protect yourself [professionally], because these are cancer cases."

The third objective of the project was to ease the recruitment and retention of surgeons in remote regions. During our interviews, we encountered at least one case of recruitment and one case of retention. Telepathology is perceived by our respondents as having positive impacts on an institution's ability to retain and recruit specialists. As two surgeons working in remote hospitals noted:

"Telepathology ensures that the pathology service is maintained, which is essential for surgeons and specialists. There are more specialties in hospitals that have pathologists than in those that don't. Telepathology should therefore help us keep specialists here, such as gynecologists. When you're thinking of putting down roots somewhere, you can be sure that whether or not the hospital has a pathologist is part of the equation."

"It's our luck that we have access to telepathology. In my case, if we didn't have it, I definitely wouldn't have come to work here. I am an oncologist surgeon, so I simply wouldn't have."

With the increasing complexity of medicine in general, and pathology in particular, one pathologist explained that such specialization runs the risk of contributing even more to the current shortage of pathologists in remote regions, justifying the continuous investments in telepathology:

"Young pathologists are specializing more and more. I'm a product of the 90s, a time when we did everything. Nowadays, pathologists are often better in one specific branch within our field. In this context, sending a pathologist to a remote region is not very appealing."

For technologists, it appears that telepathology can also add value to their day-to-day jobs:

"We play a more important role, especially since we are responsible for manipulating large specimens used during intraoperative examinations. The pathologist is at the other end of the camera, and we have the specimens in front of us; we cut them and prepare the slides that are electronically sent to the pathologist. These are new and major responsibilities that enrich our job."

For some technologists, however, telepathology is perceived as somewhat of a threat. "My fear is that one day our work will be transferred elsewhere, and then we'll be nothing more than specimen wrappers," said one technologist. In an environment characterized by 
limited resources, some technologists working in requesting sites also fear additions to their workload.

\section{Discussion}

The primary aim of this study was to better understand the actual and perceived impacts of one of the largest telepathology networks in the world. Our findings clearly reveal that telepathology is highly useful in regional hospitals that do not have a pathologist on site. Telepathology helps ensure coverage of IOCs that improve quality and access to care for populations living in remote areas. As stressed earlier, however, certain limitations inherent in the available technology mean that telepathology cannot be substituted completely for diagnoses performed under a microscope.

The benefits highlighted in this study should not leave the impression that implementing telepathology is a trivial matter. On the contrary, many problems may be encountered [8]. On a technical level, it is vital that the technology and software components perform well and are reliable once the system is up and running. When the first technical disruptions do occur, they must not be allowed to undermine user buy-in into the system. There needs to be a breaking-in phase and some experimentation before telepathology can be used for intraoperative exams. As for the post-deployment phase, our interviewees often remarked that quality technical support is another critical success factor.

From a human perspective, one should not underestimate the effort required to learn how to use the technology and the effect that this has on clinical practices. We observed deeply rooted work habits that lead to some resistance, mainly among technologists. As stressed by Meyer and Paré [8], the success of a telepathology project is often associated with the development of a relationship of trust between the various stakeholders. For example, the surgeon must trust the pathologist taking part in an intraoperative examination, and the pathologist, in turn, must trust the technologist preparing the slides or handling large specimens. In this regard, the effort required for change management should not be underestimated. Expectations also need to be well managed. Failing to do so may create an unfavourable situation. The presence of a clinical champion is another key condition for success at both the requesting sites and the responding sites.

Implementing telepathology also carries many challenges for hospital administrators such as identifying partners and signing contractual agreements between institutions, harmonizing clinical practices between partners (e.g. the cutting of specimens), building a medical laboratory (if required) and reorganizing work, both at the requesting sites and the responding sites. Several committees played key roles in implementing the telepathology project in Eastern Quebec. For instance, a project management committee was formed of a medical director for the project, a project manager and an IT specialist. The committee's main responsibilities were to plan deployment of telepathology on the area served, to coordinate and oversee collaboration on the work in accordance with the plan, to prepare a risk and change management plan and to report periodically on project progress to the ministerial authorities. During the pre-deployment phase, committee members visited each of the teams responsible for implementing telepathology in each site. As another example, 
a steering committee, made up of 18 pathologists from all the concerned regions, was also formed. The committee had a mandate to propose organizational models, draft a clinical protocol guide, advise the executive committee and the management committee, and monitor network deployment activities to ensure a coherent implementation. This committee also took part in the final selection of equipment and software, assisting in clinical and technical assessments.

To conclude, the deployment of the telepathology network in Eastern Quebec has helped to ensure continuous coverage of IOCs in regional hospitals that do not have a pathologist on site and, consequently, prevent service disruptions. Surgeons who used telepathology during IOCs believed that this approach helped them to improve the quality of care they provide to their patients, in particular by reducing the number of second surgeries and patient transfers. From an organizational perspective, telepathology has also contributed to the recruitment and retention of surgeons in remote regions. Despite these positive findings, more research is needed to investigate the challenges and benefits associated with the implementation of large and decentralized telepathology networks.

\section{Acknowledgements}

The authors would like to thank the clinicians and managers who participated to this study. The financial support of Canada Health Infoway is also acknowledged.

\section{Author Disclosure Statement}

No competing financial interests exist.

\section{References}

1. American Telemedicine Association. Clinical Guidelines for Telepathology. 2014. http://www.americantelemed.org/docs/default-source/standards/clinical-guidelinesfor-telepathologyFBFDA18D6793.pdf?sfvrsn=8. Accessed February 15, 2015.

2. Furness PN, Bamford WM. Telepathology. Current Diagnostic Pathology 2001; 7:281-291.

3. Têtu B, Evans A. Canadian licensure for the use of digital pathology for routine diagnoses: one more step toward a new era of pathology practice without borders. Archives of Pathology and Laboratory Medicine 2014; 138:302-304.

4. BCC Research. Global Markets for Telemedicine Technologies. 2012. http://www.bccresearch.com/report/telemedicine-technologies-global-marketshlc014e.html. Accessed February 13, 2015.

5. Trudel MC, Paré G, Têtu B, Sicotte C. The effects of a regional telepathology project: a study protocol. BMC Health Services Research 2012; 12:64

6. Institut de la Statistique du Québec. Population of Quebec - 1971-2014. 2015. http://www.stat.gouv.qc.ca/statistiques/population-demographie/structure/qc_197120xx.htm. Accessed April 28, 2015. 
7. Têtu B, Perron E, Louhalia S, Paré G, Trudel MC, Meyer J. The Eastern Québec telepathology network: a three-year experience of clinical diagnostic services. Diagnostic Pathology 2014; 9(Suppl 1):S1.

8. Meyer J, Paré G. Telepathology impacts and implementation challenges: a scoping review. Archives of Pathology \& Laboratory Medicine, forthcoming.

9. Dubé L, Paré G. Rigor in IS positivist case research: current practices, trends, and recommendations. MIS Quarterly 2003; 27:597-635.

10. Canada Health Infoway. Utilization and benefits evaluation indicators for telepathology. 2012; https://www.infoway-inforoute.ca/en/resource-centre/advancedsearch?q=benefits\%20evaluation. Accessed April 28, 2015.

11. Miles MB, Huberman AM. Qualitative data analysis: an expanded sourcebook. Beverly Hills CA Sage, 1994.

12. Perron E, Louahlia S, Nadeau L, Boilard F, Orain M, Têtu B. Telepathology for intraoperative consultations and expert opinions: the experience of the Eastern Quebec telepathology network. Archives of Pathology and Laboratory Medicine 2014; 138:1223-1228.

Reprint requests should be directed to the corresponding author. 